\title{
A Short Certificate-based Signature Scheme with Provable Security
}

\author{
Ying-Hao Hung, Sen-Shan Huang, Yuh-Min Tseng* \\ Department of Mathematics, National Changhua University of Education, \\ Jin-De Campus, Changhua 500, Taiwan, R.O.C. \\ e-mail: ymtseng@cc.ncue.edu.tw \\ crossref http://dx.doi.org/10.5755/j01.itc.45.3.12814
}

\begin{abstract}
Certificate-based signature (CBS) is an attractive paradigm since it simultaneously solves the certificate revocation problem in conventional signatures and the key escrow problem in ID-based signatures. In particular, short certificate-based signatures are useful in bandwidth reduction for communication due to their short signature lengths. However, it is still a challenging and open problem to design a secure short certificate-based signature (SCBS) scheme. Recently, to solve this problem, Li et al. proposed an efficient SCBS scheme. However, in this article, we will show that $\mathrm{Li}$ et al.'s scheme is insecure against Type I adversary (i.e. uncertified entity) under an accredited security model. Moreover, we propose a new SCBS scheme with provable security. Based on the computational Diffie-Hellman (CDH) assumption, we demonstrate that our SCBS scheme possesses existential unforgeability against adaptive chosen-message attacks under the same accredited security model. When compared with previous SCBS schemes, our scheme is the first provably secure SCBS scheme while retaining efficiency.
\end{abstract}

Keywords: Short signature; Certificate-based signature; Existential unforgeability.

\section{Introduction}

In conventional public-key system (PKS) settings $[1,2]$, a trusted certificate authority $(\mathrm{CA})$ issues public key certificates to provide un-forgeable and trusted links between the identities and the public keys of the users. Therefore, a public key infrastructure (PKI) is required to manage and maintain certificates of all the users. In such a system, anyone who wants to verify signatures of other entities must verify their authorized public key certificates beforehand to ensure that these public keys are still valid. Hence, the certificate management is generally considered to be costly, when adopted in a PKI.

To simplify the certificate management, Shamir [3] introduced the concept of identity (ID)-based publickey system (ID-PKS) setting. Until 2001, Boneh and Franklin [4] proposed the first practical ID-PKS setting and ID-based encryption (IBE) scheme. In their ID-PKS setting, a user's public key is determined by some identity information such as social security number, e-mail address, and name. This avoids the necessity of certificates, and associates an implicitly verified public key to each user. A trusted private key generator (PKG) with a master secret key is responsible to generate and send each user a private key via a secure channel. However, since the PKG knows each user's private key, it can generate signatures on behalf of any user, or decrypt any ciphertexts sent to any user. Hence, the key escrow property becomes an inherent problem in the ID-PKS setting, so that ID-PKS setting is only suitable for a closed organization where the PKG is fully trusted by everyone in the group.

To solve the key escrow problem in the ID-PKS setting, Al-Riyami and Paterson [5] presented a new paradigm, called certificateless PKS (CL-PKS) setting, which eliminates the usage of certificates in the conventional PKS settings. In the CL-PKS setting, there are two roles, namely, the key generation center (KGC) and users. A user independently generates a public/secret key pair, and the KGC generates a partial private key in accordance with the identity of the user and then sends it to the user via a secure channel. To decrypt a message, a user requires both her/his secret key and the associated partial private key. Note that the KGC does not know any user's secret key so that it is unable to impersonate a user or decrypt the ciphertexts sent to a user. Therefore, the CL-PKS setting solves the key escrow problem in the ID-PKS setting by eliminating the usage of certificates in the conventional PKS setting. However, due to the lack of public key certificates, both the ID-PKS and CL-PKS settings must provide additional revocation

\footnotetext{
* Corresponding author
} 
mechanisms [6-9]. In 2003, Gentry [10] introduced the notion of certificate-based PKS (CB-PKS) setting, which resolves the inherent key escrow problem in ID-PKS setting and the certificate revocation problem in the conventional PKS. In Gentry's scheme, a certificate acts as a partial private key as well. A user independently generates her/his public/secret key pair and sends the public key to a trusted certificate authority (CA). Then the CA generates a certificate for the user by the user's public key and some additional identity information

Table 1. Comparisons among the conventional PKS, ID-PKS, CL-PKS and CB-PKS settings

\begin{tabular}{ccccc}
\hline & PKS & ID-PKS & CL-PKS CB-PKS \\
{$[\mathbf{1 , 2}]$} & {$[\mathbf{4}]$} & [5] & {$[\mathbf{1 0 ]}$} \\
\hline $\begin{array}{c}\text { Averting key } \\
\text { escrow problem }\end{array}$ & Yes & No & Yes & Yes \\
\hline $\begin{array}{c}\text { Required channel } \\
\text { for revocation }\end{array}$ & Public & secure & secure & Public \\
\hline $\begin{array}{c}\text { Level of trust } \\
\text { placed on the } \\
\text { CA/PKG/KGC }\end{array}$ & Low & High & Middle & Low \\
\hline $\begin{array}{c}\text { Certificate } \\
\text { validation before } \\
\text { encrypting and } \\
\text { verifying }\end{array}$ & Required & $\begin{array}{c}\text { Not } \\
\text { required }\end{array}$ & $\begin{array}{c}\text { Not } \\
\text { required }\end{array}$ & $\begin{array}{c}\text { Not } \\
\text { required }\end{array}$ \\
\hline
\end{tabular}

Since then, the CA updates the certificate periodically. To sign a message or decrypt a ciphertext, the user requires both her/his secret key and an up-to-date certificate. Since the CA is unable to obtain the secret key of any user, the key escrow problem will not take place in the CB-PKS setting. Meanwhile, CB-PKS setting also solves the certificate revocation problem.

Table 1 lists the comparisons among the conventional PKS [1, 2], ID-PKS [4], CL-PKS [5], and CB-PKS [10] settings in terms of averting the key escrow problem, revocable functionality, the level of trust placed on the $\mathrm{CA} / \mathrm{PKG} / \mathrm{KGC}$ and certificate validation before encrypting and verifying. It is obvious that the ID-PKS setting suffers from the key escrow problem. For the level of trust placed on the $\mathrm{CA} / \mathrm{PKG} / \mathrm{KGC}$, the CB-PKS setting is better than the others. Except for the conventional PKS and the CBPKS settings, the other settings remove the need of certificates. In the conventional PKS settings, the users can verify illegal or compromised users by referring to the certificate revocation list (CRL). In the ID-PKS and CL-PKS settings, the PKG/KGC adopts a secure channel to transmit the private keys to non-revoked users periodically $[4,5]$. In the CB-PKS setting, the CA updates the certificates via a public channel. According to Table 1, the CB-PKS construction possesses the advantages of both ID-PKS (implicit certification) and conventional PKS (no escrow) settings while it does not need a secure channel for revocation. In the conventional PKS setting, before verifying a signature by the signer's public key, one needs to verify the signer's certificate issued by the CA to guarantee the validation of the signer's public key. Due to additional computation time and storage, the certificates in conventional PKS settings are costly to use and manage.

\subsection{Motivation}

Digital signature is one important cryptographic primitive, which provides the integrity, authentication and non-repudiation of messages. Indeed, authentication (identification) schemes [11-15] may be implemented by employing signature schemes. With the rapid growth of wireless communications, clients (users) often use handheld wireless devices to access remote servers via open network channels. When cryptographic mechanisms are involved in wireless environments, these wireless devices are generally resource-constrained because they possess low-power energy and limited computing capability. In this case, numerous data bits of communication and cryptographic operations with expensive computations would become heavy load for wireless devices. Hence, it is a critical issue to alleviate the communication and computational load of wireless devices. For wireless devices such as smart card, PDA, cell phone, RFID chip and sensor, message communication consumes more time and energy than computation does. To transmit one bit of data requires more energy than to execute one 32-bit instruction [16]. Therefore, reducing the number of communication bits becomes an important issue for cryptographic mechanisms executed on wireless devices.

Since the usage of short signature aims at the reduction of communication bandwidth, it is suitable for wireless environments. In 2001, Boneh et al. [17] constructed the first short signature scheme from bilinear pairings in conventional PKS setting. It is half the size of a DSA signature with a similar level of security. Afterwards, several concrete short signature schemes $[18,19]$ were proposed in the standard model (without random oracles). On the other hand, several researchers [20-23] also proposed short certificateless signature schemes in CL-PKS setting. However, it is still a challenging and open problem to design a secure short certificate-based signature (SCBS). Recently, to solve this problem, Li et al. [24] proposed an efficient SCBS scheme. However, in this article, we will show that Li et al.'s scheme is insecure against Type I adversary (i.e. uncertified entity). Moreover, we will propose the first provably secure SCBS scheme.

\subsection{Related work}

Following Gentry's [10] concept, in 2004, Kang et al. [25] proposed the first certificate-based signature (CBS) scheme which is derived from bilinear pairings on elliptic curves. In 2007, Li et al. [26] introduced the key replacement attack on the CB-PKS setting and demonstrated Kang et al.'s scheme is insecure against key replacement attacks. Since the key replacement 
attacks might occur in the CB-PKS setting, $\mathrm{Li}$ et al. [26] redefined Kang et al.'s security model by additionally addressing key replacement attacks and proposed an improved version. In 2008, Liu et al. [27] furthermore proposed two new CBS schemes. One of them did not require any pairing operations in the random oracle model and the other was secure without random oracle. Unfortunately, Zhang et al. [28] showed that Liu et al.'s first scheme was insecure and proposed an improved scheme. Meanwhile, $\mathrm{Wu}$ et al. [29] also proposed an improved CBS scheme. However, the signature sizes of all the CBS schemes mentioned above are more than one group element.

In 2011, Liu et al. [30] proposed the first short certificate-based signature (SCBS) scheme. Unfortunately, Cheng et al. [31] showed that their scheme is insecure against a Type I adversary under an accredited security model defined in [26-28]. Recently, Li et al. [24] proposed a new SCBS scheme. However, the security model of Li et al.'s scheme is weaker than the accredited security model [26-28] in the sense that a Type I adversary (i.e. uncertified entity) can extract neither singer's secret key nor certificate of a target entity. Hence, it is still a challenging and open problem to design a secure SCBS scheme under the accredited security model.

\subsection{Contribution}

In this article, we first show that Li et al.'s SCBS scheme [24] is insecure against a Type I adversary under the accredited security model in [26-28]. To achieve our goal, we redefine the framework of SCBS schemes, in which the public key of a user is determined by both the user and the CA. In the accredited security model [26-28], an adversary can extract either a singer's secret key or the associated certificate of a target entity. Finally, we propose the first provably secure SCBS signature scheme in the random oracle model $[32,33]$. Our scheme has the following features. Firstly, as compared with the previously proposed CBS and SCBS schemes, our scheme enjoys lower communication bandwidth while retaining computation efficiency. Secondly, the proposed scheme possesses existential unforgeability against adaptive chosen-message attacks under the computational Diffie-Hellman $(\mathrm{CDH})$ assumption. Lastly, to the best of our knowledge, our scheme is the first provable secure SCBS scheme under the accredited security model in [26-28].

The remainder of the article is organized as follows. In Section 2, we give a brief introduction for bilinear pairings and security assumption. In Section 3, we redefine the framework and security notions for SCBS schemes. In section 4, we review Li et al.'s SCBS scheme and show how a Type I adversary can successfully attack their scheme. Our concrete SCBS scheme is given in Section 5. In Section 6, we analyze the security of our scheme. Comparisons are demonstrated in Section 7. Finally, we draw a conclusion in Section 8.

\section{Preliminaries}

In this section, we briefly present some properties of bilinear pairings and a relevant security assumption [4].

\subsection{Bilinear pairings}

Let $G$ and $G_{T}$ be additive and multiplicative cyclic groups of the same prime order $q$, respectively. An admissible bilinear pairing is a map $\hat{e}: G \times G \rightarrow G_{T}$ with the following properties:

1. Bilinearity: for $P, Q \in G$ and $a, b \in Z_{q}{ }^{*}$, the map $\hat{e}$ satisfies the equality $\hat{e}(a P, b Q)=\hat{e}(P, Q){ }^{a b}$.

2. Non-degeneracy: there exist $P, Q \in G$ such that $\hat{e}(P, Q) \neq 1$.

3. Computability: for all $P, Q \in G, \hat{e}(P, Q)$ can be computed efficiently.

\subsection{Security assumption}

Here, we present a hard mathematical problem and define its corresponding assumption.

- Computational Diffie-Hellman (CDH) problem: given $P, a P, b P \in G$ with uniformly random choices of $a, b \in Z_{q}{ }^{*}$, the $\mathrm{CDH}$ problem is to compute $a b P$.

Definition 1. The $\mathrm{CDH}$ assumption in $G$ is defined as follows. Given $P, a P, b P \in G$ with uniformly random choices of $a, b \in Z_{q}{ }^{*}$, there exists no probabilistic polynomial-time adversary (PPT) $\mathcal{A}$ with nonnegligible probability who can compute $a b P$. The successful probability (advantage) of the adversary $\mathcal{A}$ is presented as

$$
\operatorname{Adv}_{\mathcal{A}}=\operatorname{Pr}\left[\mathcal{A}(P, a P, b P)=a b P: P \in G, a, b \in Z_{q}^{*}\right] .
$$

\section{Framework and adversarial model of SCBS}

\subsection{Framework}

We present the framework of short certificatebased signature (SCBS) schemes depicted in Fig.1, which is modified from that of the CBS scheme in [26-28]. Our framework is slightly different from that of the conventional CBS schemes in the sense that the full public key of a user is generated by both the user and the CA. A SCBS scheme is specified by five algorithms, namely, Setup, User key generation, Certificate generation, Sign and Verify algorithms.

- Setup is a probabilistic algorithm run by the CA that takes a security parameter as input, and returns a master secret key and public parameters $P P . P P$ is made public and available for all the other algorithms.

- User key generation is a probabilistic algorithm run by a user that takes as input the user's identity 
$I D$, and outputs the secret key $S_{I D}$ and the partial public key $P_{I D}$.

- Certificate generation is a probabilistic algorithm run by the CA that takes as input the master secret key, the public parameters $P P$, a user's identity $I D$ and partial public key $P I D$, and returns the user's certificate $C_{I D}$ and (full) public key $P K_{I D}=\left(P_{I D}, R_{I D}\right)$ to the user, and publishes $(I D$, $\left.P K_{I D}\right)$ in a public directory.

- Sign is a deterministic algorithm run by a user that takes as input the user's secret key $S_{I D}$, certificate $C_{I D}$ and a message $M$, and returns a signature $\sigma$.

- Verify is a deterministic algorithm that takes as input a message $M$, a signature $\sigma$, a user's identity $I D$ with the public key $P K_{I D}$, and outputs either "accept" or "reject".

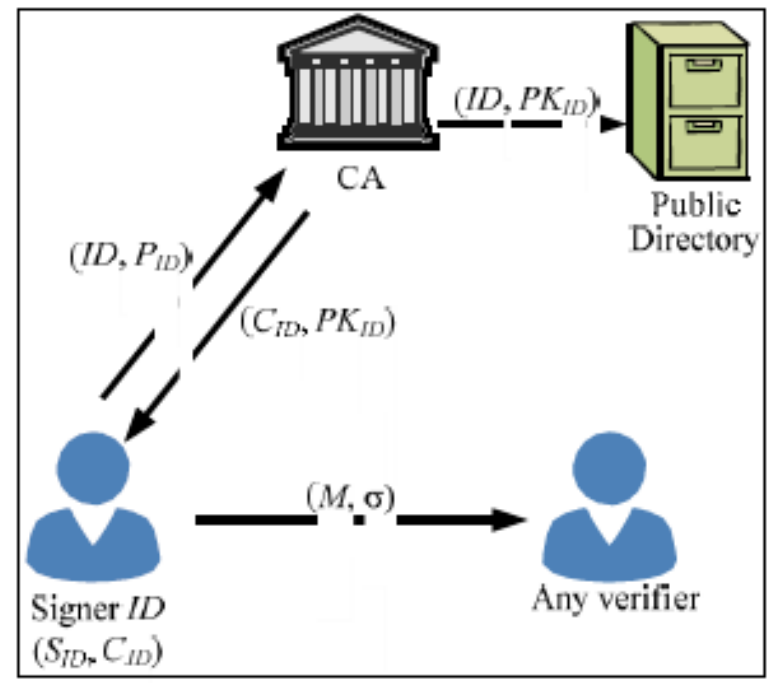

Figure 1. Framework of SCBS schemes

\subsection{Security model}

Based on the security model of CBS schemes in [26-28], we define "existential unforgeability of short certificate-based signatures against adaptive chosenmessage attacks" (UF-SCBS-ACM). In UF-SCBSACM attacks, the security notions for SCBS schemes include two types of adversaries, namely, Type I and Type II adversaries with different query capabilities. A Type I adversary $A_{I}$ acts as an uncertified entity who does not have access to the master secret key $s$ so that it cannot obtain the certificate of a target entity. A Type II adversary $A_{I I}$ models the malicious CA who owns the master secret key $s$, but cannot obtain the secret key of the target entity. The security notions for SCBS schemes are modeled by the following two games (Games 1 and 2) between a challenger $C$ and the two types of adversaries.

Definition 2. (UF-SCBS-ACM). A SCBS scheme offers existential unforgeability against adaptive chosen-message (UF-SCBS-ACM) attacks if no PPT adversary $\mathcal{A}$ of Type I or Type II has a non- negligible advantage in the following two games (Games 1 and 2) played between a challenger $C$ and the adversary $\mathcal{A}$.

\section{Game 1 (for Type I adversary, AI)}

- Setup. The challenger $C$ runs the Setup algorithm to produce a master secret key $s$ and a list of public parameters $P P$. PP is given to $A_{I}$ and $s$ is kept secret by $C$.

- Queries. The adversary $A_{I}$ may issue a number of different queries to the challenger $C$ in an adaptive manner as follows.

- User key generation (ID). The challenger $C$ runs the User key generation algorithm to return the user's partial public key $P_{I D}$ to $A_{I}$.

- Certificate generation (ID, $P I D)$. The challenger $C$ runs the Certificate generation algorithm to return the user's certificate $C_{I D}$ and public key $P K_{I D}=\left(P_{I D}, R_{I D}\right)$ to $A_{I}$.

- Corruption (ID). Upon receiving this query, the challenger $C$ returns the user's secret key $S_{I D}$ to A.

- Public key replacement (ID, PK'ID). The adversary $A_{I}$ chooses a new public key $P K_{I D}^{\prime}=\left(P^{\prime} I D, R_{I D}^{\prime}\right)$ for the user with identity $I D$. The challenger $C$ records this replacement.

- $\quad \operatorname{Sign}(m, I D)$. Upon receiving this query on $(m$, $I D)$ under the public key $P K_{I D}=\left(P I D, R_{I D}\right)$, the challenger $C$ generates a valid signature $\sigma$ and returns it to $A_{I}$ even though the challenger $C$ does not hold the secret key and the certificate of the user with identity $I D$.

- Forgery. The adversary $A_{I}$ generates a signature tuple $\left(m^{*}, \sigma^{*}, I D^{*}, P K_{I D^{*}}\right)$, where $P K_{I D^{*}}$ is the original public key without replacement. The advantage of the adversary $A_{I}$ is defined as the probability that $A_{I}$ wins Game 1 . We say that the adversary $A_{I}$ wins Game 1 if the following conditions are all satisfied.

1. The response of the Verification algorithm on $\left(m^{*}, \sigma^{*}, I D^{*}, P K_{I D^{*}}\right)$ is "accept".

2. $\left(m^{*}, I D^{*}\right)$ has never been submitted during the sign query.

3. $I D^{*}$ has never been submitted during the Certificate generation query.

\section{Game 2 (for Type II adversary, AII)}

- Setup. The challenger $C$ runs the setup algorithm to produce a master secret key $s$ and a list of public parameters $P P$. $P P$ and $s$ are sent to $A I I$.

- Queries. Since the adversary $A_{I I}$ knows the master secret key, it can compute the certificates and the public keys of all the users. The adversary $A_{I I}$ may make a number of queries as in Game 1, except the 
certificate generation query because it can compute the certificates of all the users.

- Forgery. The adversary $A_{I I}$ generates a signature tuple $\left(m^{*}, \sigma^{*}, I D^{*}, P K_{I D^{*}}\right)$, where $P K_{I D^{*}}$ is the original public key without replacement. The advantage of the adversary $A_{I I}$ is defined as the probability that $A_{I I}$ wins Game 2 . We say that the adversary $A_{I I}$ wins Game 2 if the following conditions are all satisfied.

1. The response of the Verification algorithm on $\left(m^{*}, \sigma^{*}, I D^{*}, P K_{I D}\right)$ is "accept".

2. $\left(m^{*}, I D^{*}\right)$ has never been submitted during the sign query.

3. $I D^{*}$ has never been submitted during the Corruption query.

\section{Review and weakness of Li et al.'s SCBS scheme}

Recently, Li et al. [24] presented a SCBS scheme and claimed their scheme is secure against both Type I and Type II adversaries in the random oracle model. However, the security model of Li et al.'s scheme is weaker than the accredited security model in [26-28]. In their model, a Type I adversary (i.e. uncertified entity) extracts neither the secret key nor the certificate of a target entity. On the contrary, the accredited security model allows an adversary to obtain either the secret key (Type I adversary) or the certificate (Type II adversary) of a target entity as described in Games 1 and 2 in Section 3. In this section, we show that their scheme is insecure in the presence of Type I adversary under the accredited security model. In the following, we first review Li et al.'s SCBS scheme.

\subsection{Li et al.'s SCBS scheme}

Li et al.'s SCBS scheme consists of five algorithms:

- Setup: Given a security parameter $1^{k}$, the CA generates a list of public parameter $P P=<G, G T, e$, $q, P, Q, m p k, H_{0}, H_{1}>$ by performing the following steps.

1. Let $\left(G, G_{T}\right)$ be a bilinear group pair of a prime order $q$, and let $e: G \times G \rightarrow G_{T}$ be a bilinear map.

2. Select a random number $s \in Z_{q}{ }^{*}$ and two random elements $P, Q \in G$. Set $s$ as the master secret key $m s k$, and $s \cdot P$ as the system public key $m p k$.

3. Choose two cryptographic hash functions $H_{0}:\{0,1\}^{*} \times G \times G \rightarrow G_{T}$ and $H_{1}:\{0,1\}^{*}$ $\rightarrow Z_{q}^{*}$.

- User key generation: Given PP, a user with identity $I D$ randomly selects a secret key $s_{I D} \in Z_{q}{ }^{*}$ and computes the corresponding public key
$P K_{I D}=\left(P K_{I D}^{1}, P K_{I D}^{2}\right)$, where $P K_{I D}^{1}=S I D \cdot P$ and $P K^{2}{ }_{I D}=S_{I D} \cdot Q$.

- Certificate generation: Given $P P$, the master secret key $s$, a public key $P K_{I D}$ of the user with identity $I D$, the CA computes $Q_{I D}=H_{0}\left(I D, P K_{I D}\right)$ and the certificate $\operatorname{Cert}_{I D}=s \cdot Q_{I D}$.

- Sign: Taking as input $P P, I D$, a secret key $S I D$, a certificate $\operatorname{Cert}_{I D}$ and a message $m \in\{0,1\}^{*}$, a user calculates the signature $\sigma=\left(1 /\left(H_{1}(m)+s_{I D}\right)\right) \cdot \operatorname{Cert}_{I D}$.

- Verify: Given a signature pair $(m, \sigma)$, the public parameter $P P$, an identity $I D$ and a public key $P K_{I D}=\left(P K_{I D}^{1}, P K_{I D}^{2}\right)$, a verifier calculates $Q_{I D}$ $=H_{0}\left(I D, P K_{I D}\right)$. This algorithm outputs "accept" if both equalities $e\left(\sigma, H_{1}(m) P+P K_{I D}^{1}\right)=e\left(m p k, Q_{I D}\right)$ and $e\left(P K_{I D}^{1}, Q\right)=e\left(P K_{I D}^{2}, P\right)$ hold. Otherwise, it outputs "reject".

\subsection{Weakness of Li et al.'s SCBS scheme}

Here, we point out that $\mathrm{Li}$ et al.'s scheme is not secure against Type I adversary $A_{I}$ in the accredited security model of CBS schemes [26-28]. In Game 1, AI cannot issue the Certificate generation query on a target entity with identity $I D^{*}$, but can obtain the associated secret key $S_{I D} *$ by making the Corruption query on $I D^{*}$. Then, we show that $A_{I}$ can obtain both the certificate $\operatorname{Cert}_{I D}$ * and the associated secret key $S_{I D}$ * of the target entity by the following steps.

1. First, $A_{I}$ makes the User key generation query on a target user with identity $I D^{*}$ to obtain the user's public key $P K^{*}{ }_{I D}=\left(s_{I D^{*} \cdot P} \cdot s_{\left.I D^{*} \cdot Q\right)}\right.$.

2. Next, $A_{I}$ issues the Corruption query to obtain the user's secret key $S_{I D}{ }^{*}$. Then $A_{I}$ makes the Sign query to obtain a valid signature $\sigma^{*}=\left(1 /\left(H_{1}\left(m^{*}\right)+s_{I D} *\right) \cdot\right.$ Cert ID $^{*}$ on the message $m$ with respect to the public key $P K^{*}{ }_{I D}$.

3. Finally, $A_{I}$ can obtain the certificate CertID $^{*}=\sigma^{*} /\left(1 / H_{1}\left(m^{*}\right)+s_{I D}\right)$.

By above, $A_{I}$ can generate valid signatures on any messages on behalf of the target user with identity $I D^{*}$. Therefore, $A_{I}$ can win the Game 1 so that Li et al.'s SCBS scheme is insecure under the aforementioned security model. Indeed, Cheng et al. [31] have presented the same attack on Liu et al.'s SCBS scheme [30] and pointed out that it is still a challenging and open problem to design a secure SCBS scheme.

\section{Our SCBS scheme}

The proposed SCBS scheme consists of five algorithms, namely, Setup, User key generation, Certificate generation, Sign and Verify algorithms. 
- Setup: Given a security parameter $l$, the CA first generates a bilinear group pair $\left(G, G_{T}\right)$ of a prime order $q>2^{l}$, an admissible bilinear map $\hat{e}: G \times G$ $\rightarrow G_{T}$, and an arbitrary generator $P$ of $G$. Next, the CA randomly chooses a master secret key $s \in Z_{q}{ }^{*}$ and sets $P_{p u b}=s \cdot P$ as the system public key.

The CA picks three hash functions $f:\{0,1\}^{*}$ $\rightarrow Z_{q}^{*}, H_{1}$ and $H_{2}:\{0,1\}^{*} \rightarrow G$. A list of public parameters are presented as $P P=<G, G T, q, \hat{e}, P, P_{p u b}$, $f, H_{1}, H_{2}>$.

- User key generation: A user with identity ID randomly selects a secret key $S_{I D} \in Z_{q}{ }^{*}$ and sets $P_{I D}=S_{I D} \cdot P$ as her/his partial public key.

- Certificate generation: Given a user's identity ID and partial public key $P I D$, the CA randomly chooses $r_{I D} \in Z_{q}{ }^{*}$, and then computes $R_{I D}=r_{I D} \cdot P$, $h=f\left(I D, P_{I D}, R_{I D}, P_{p u b}\right)$ and the corresponding certificate $C_{I D}=r_{I D}+s \cdot h$. The CA returns the certificate $C_{I D}$ and the public key $P K_{I D}=\left(P I D, R_{I D}\right)$ to the user.

- Sign: To sign a message $m \in\{0,1\}^{*}$, the signer with a secret key $S_{I D}$ and a certificate $C_{I D}$ computes $U_{1}=H_{1}\left(m, I D, P K_{I D}, P_{p u b}\right)$ and $U_{2}=H_{2}(m, I D$, $\left.P K_{I D}, P_{p u b}\right)$ and generates the corresponding signature $\sigma=S_{I D} \cdot U_{1}+C_{I D} \cdot U_{2}$.

- Verify: Given a signature tuple $\left(m, \sigma, I D, P K_{I D}\right)$ with $P K_{I D}=\left(P_{I D}, R_{I D}\right)$, a verifier performs the following steps to validate the signature tuple:

1. Compute $h=f\left(I D, P_{I D}, R_{I D}, P_{p u b}\right), U_{1}=H_{1}(m$, $\left.I D, P K_{I D}, P_{p u b}\right)$ and $U_{2}=H_{2}\left(m, I D, P K_{I D}\right.$, $P_{p u b)}$.

2. Verify the equality $\hat{e}(P, \sigma)=\hat{e}\left(P_{I D}\right.$, $\left.U_{1}\right) \cdot \hat{e}\left(R_{I D}+h \cdot P_{p u b}, U_{2}\right)$. If it holds, output "accept". Otherwise, output "reject". Here, the correctness of the equality in Step (2) of the Verify algorithm follows since

$$
\begin{aligned}
& \hat{e}(P, \sigma)=\hat{e}\left(P, S_{I D} \cdot U_{1}+C_{I D} \cdot U_{2}\right) \\
& =\hat{e}\left(P, S_{I D} \cdot U_{1}\right) \cdot \hat{e}\left(P, C_{I D} \cdot U_{2}\right) \\
& =\hat{e}\left(S_{I D} \cdot P, U_{1}\right) \cdot \hat{e}\left(P,(r I D+s \cdot h) \cdot U_{2}\right) \\
& =\hat{e}\left(S_{I D} \cdot P, U_{1}\right) \cdot \hat{e}\left((r I D+s \cdot h) \cdot P, U_{2}\right) \\
& =\hat{e}\left(P_{I D}, U_{1}\right) \cdot \hat{e}\left(R_{I D}+h \cdot P_{p u b}, U_{2}\right) .
\end{aligned}
$$

\section{Security analysis}

In this section, we give the security analysis of the proposed SCBS scheme. Theorems 1 and 2, respectively, show that the proposed scheme is secure against Type I and Type II adversaries in the UF-SCBSACM games (Games 1 and 2) presented in Definition 3.

Theorem 1. Suppose that the hash functions $H_{1}, H_{2}$ and $f$ are random oracles. Assume that a Type I UF-SCBS-ACM adversary $\mathcal{A}$ can break the proposed SCBS scheme with non-negligible advantage $\varepsilon$ within running time $\tau$. Then, there exists an algorithm Cto solve the $\mathrm{CDH}$ problem with a non-negligible advantage

$$
\varepsilon^{\prime} \geq\left(1-1 / q_{c}\right)\left(1-1 /\left(1+q_{s}\right)\right)^{q_{s}}\left(1 /\left(q_{c}\left(1+q_{s}\right)\right)\right) \varepsilon
$$

within running time $\tau^{\prime}=\tau+O\left(q_{c}+q_{s}+q_{u}+q_{1}+q_{2}\right) \tau_{1}$, where $\tau_{1}$ is the time to perform a scalar multiplication in $G$. Also, $q_{c}, q_{s}, q_{u}, q_{1}$, and $q_{2}$, denote, respectively, the maximal numbers of $\mathcal{A}$ 's queries to the Certificate generation oracle, the Sign oracle, the User key generation oracle, the random oracle $H_{1}$, and the random oracle $H_{2}$. $\mathcal{A}$ can, respectively, ask $q_{c}$ queries to the Certificate generation oracle, $q_{1}$ queries to the random oracle $H_{1}$, $q 2$ queries to the random oracle $H_{2}, q_{u}$ queries to the User key generation oracle, and $q_{s}$ queries to the Sign oracle.

Proof. Assume that an algorithm $C$ is given a random instance $(P, a P, b P)$ of the $\mathrm{CDH}$ problem, where $P$, $a P, b P \in G$ with unknown $a, b \in Z_{q}{ }^{*}$. Let $J=a b P$ be the solution of the $\mathrm{CDH}$ problem. The algorithm $C$ finds $J$ by interacting with the adversary $\mathcal{A}$ as follows.

- Setup. The challenger $C$ creates a list of public parameters $P P=\left\{G, G T, q, \hat{e}, P, P_{p u b}, f, H_{1}, H_{2}\right\}$ by setting $P_{p u b}=a P$. The challenger $C$ gives $\mathcal{A}$ the public parameters $P P$. Here, the hash functions $f$, $H_{1}$ and $H_{2}$ are random oracles controlled by the challenger $C$. To be consistent and collision-free, the challenger $C$ maintains four initially empty lists $L_{f}, L_{1}, L_{2}$, and $L_{C}$ to record the responses of the query oracles.

- Queries: The challenger $C$ first randomly chooses $t \in\left[1, q_{c}\right]$ and lets $I D^{\prime}$ denote the target identity of the $t$-th query to the Certificate generation oracle. The challenger $C$ answers the queries issued by $\mathcal{A}$ as follows.

- User key generation (ID): C maintains an initially empty list $P^{\text {list }}$ of tuples $\left(I D, P I D, S_{I D}\right)$. If $I D$ 's corresponding tuple $\left(I D, P_{I D}, S_{I D}\right)$ exists in $P^{\text {list }}$, $C$ return $P_{I D}$ as answer. Otherwise, $C$ randomly chooses a value $S_{I D} \in Z_{q}{ }^{*}$, computes $P_{I D}=S_{I D} \cdot P$, returns $P_{I D}$ to the adversary $\mathcal{A}$, and adds $(I D, P I D$, $S_{I D)}$ to $P^{\text {list }}$. Additionally, if $I D=I D^{\prime}$, the challenger $C$ randomly chooses $r I D \in Z_{q}^{*}$, sets $R_{I D}=r_{I D} \cdot P$, and stores the tuple $\left(I D, P_{I D}, R_{I D}\right.$, $\perp)$ in the initially empty list $L_{C}$.

- $\quad f\left(I D, P_{I D}, R_{I D}\right): C$ maintains an initially empty list $L_{f}$ of tuples $\left(I D, P_{I D}, R_{I D}, h\right)$. If there exists a tuple $\left(I D, P_{I D}, R_{I D}, h\right)$ in $L_{f}$ related to the query $f\left(I D, P_{I D}, R_{I D}\right), C$ returns $h$ as answer. Otherwise, $C$ randomly chooses $h \in Z_{q}{ }^{*}$ and 
returns it to the adversary $\mathcal{A}$. Then, the challenger $C$ adds (ID, $\left.P_{I D}, R_{I D}, h\right)$ to $L_{f}$.

- $\quad H_{1}\left(m, I D, P K_{I D}\right): C$ maintains an initially empty list $L_{1}$ of tuples $\left(m, I D, P K_{I D}, w, U_{1}\right)$. If there exists a tuple $\left(m, I D, P K_{I D}, w, U_{1}\right)$ in $L_{1}$ related to the query $H_{1}\left(m, I D, P K_{I D}\right), C$ returns $U_{1}$ to $\mathcal{A}$. Otherwise, $C$ randomly chooses $w \in Z_{q}{ }^{*}$, sets $U_{1}=w \cdot P$, returns $U_{1}$ to $\mathcal{A}$, and adds $\left(m, I D, P K_{I D}\right.$, $\left.w, U_{1}\right)$ to $L_{1}$.

- $\quad H_{2}\left(m, I D, P K_{I D}\right): C$ maintains an initially empty list $L_{2}$ of tuples $\left(m, I D, P K_{I D}, u, U_{2}\right.$, coin $)$. If there exists a tuple $\left(m, I D, P K_{I D}, u, U_{2}\right.$, coin $)$ in $L_{2}$ related to the query $H_{2}\left(m, I D, P K_{I D}\right), C$ returns $U_{2}$ to $\mathcal{A}$. Otherwise, $C$ randomly chooses $u \in Z_{q}^{*}$. If $I D \not I D^{\prime}, C$ sets $U_{2}=u \cdot P$ and coin $=0$. If $I D=I D^{\prime}, \quad C$ first tosses a coin $\in\{0,1\}$ with $\operatorname{Pr}[$ coin $=1]=\delta$ for some $\delta$ that will be determined later. Moreover, $C$ sets $U_{2}=u \cdot P$ if $\operatorname{coin}=0$, and $U_{2}=u \cdot b P$ if coin $=1$. Then the challenger $C$ returns $U_{2}$ to the adversary $\mathcal{A}$ and adds $(m, I D$, $P K_{I D}, u, U_{2}$, coin) to the list $L_{2}$.

- Certificate generation (ID, PID): $C$ maintains an initially empty list $L_{C}$ of tuples $\left(I D, P_{I D}, R_{I D}\right.$, $\left.C_{I D}\right)$. If there exists $\left(I D, P_{I D}, R_{I D}, C_{I D}\right)$ in the list $L_{C}, C$ returns the certificate $C_{I D}$ and public key $P K_{I D}=\left(P_{I D}, R_{I D}\right)$ to $\mathcal{A}$. Otherwise, the challenger $C$ performs the following procedures. If $I D=I D^{\prime}, C$ returns failure and terminates. If $I D \neq I D^{\prime}$, the challenger $C$ chooses two random elements $v, h \in Z_{q}{ }^{*}$, and sets $C_{I D}=v$, $R_{I D}=v \cdot P-h \cdot P_{p u b}$, and $h=f\left(I D, P_{I D}, R_{I D}\right)$. If this particular tuple $\left(I D, P_{I D}, R_{I D}, h\right)$ already exists in $L_{f}, C$ discards it, chooses two new random elements $v, h \in Z_{q}{ }^{*}$, and repeats the process until the outcome $\left(I D, P_{I D}, R_{I D}, h\right)$ is new to $L f$. Finally, $C$ adds (ID, $\left.P_{I D}, R_{I D}, C_{I D}\right)$ to the list $L_{C}$ and returns $C_{I D}$ to the adversary $\mathcal{A}$.

- Corruption (ID): If ID's related tuple (ID, $P_{I D}$, $S_{I D)}$ already exists in $P^{\text {list }}, C$ returns $S_{I D}$ as answer. Otherwise, $C$ issues User key generation query on $I D$ and returns the outcome $S_{I D}$ to A.

- $\quad$ Public key replace (ID, $\left.P K^{\prime} I D\right)$ : The adversary $A_{I}$ chooses a new public key $P K_{I D}^{\prime}=\left(P_{I D}^{\prime}, R_{I D}^{\prime}\right)$ of a user with identity $I D$ and sends it to the challenger $C$. Then $C$ replaces $I D$ 's corresponding tuples $\left(I D, P_{I D}, S_{I D}\right)$ in $P^{\text {list }}$ and $\left(I D, P_{I D}\right.$, $\left.R_{I D}, C_{I D}\right)$ in $L_{C}$, respectively, with the new tuples $\left(I D, P^{\prime} I D, \perp\right)$ and $\left(I D, P^{\prime} I D, R^{\prime} I D, \perp\right)$.

- $\quad \operatorname{Sign}(m, I D)$ : When receiving a signature query on $(m, I D)$ under the public key $P K_{I D}=q\left(P_{I D}\right.$, $R_{I D}$ ), the challenger $C$ first issues the $H_{2}$ query with ( $\left.m, I D, P K_{I D}\right)$ and obtains the corresponding tuple $\left(m, I D, P K_{I D}, u, U_{2}\right.$, coin) $L_{2}$. The challenger $C$ discusses the following two cases.

1. If $I D=I D^{\prime}$ and coin=1, $C$ returns failure and terminates.

2. Otherwise, $C$ chooses two random elements $h, w \in Z_{q}{ }^{*}$ and computes the signature $\sigma=w \cdot P_{I D}+u \cdot R_{I D}+u \cdot h \cdot P_{p u b}$. Then, $C$ adds $(I D$, $\left.P_{I D}, R_{I D}, h\right)$ in $L_{f}$ and $\left(m, I D, P K_{I D}, w, U_{1}\right)$ in $L_{1}$, and returns the signature $\sigma$. Even though the challenger $C$ does not hold the corresponding secret key and the certificate of the user with $I D$, the signature $\sigma$ is still valid because it passes the verification as follows.

$$
\begin{aligned}
& \hat{e}(P, \sigma)=\hat{e}\left(P, w \cdot P_{I D}+u \cdot R_{I D}+u \cdot h \cdot P_{p u b}\right) \\
& =\hat{e}\left(P, w \cdot P_{I D}\right) \cdot \hat{e}\left(P, u \cdot R_{I D}+u \cdot h \cdot P_{p u b}\right) \\
& =\hat{e}\left(w \cdot P, P_{I D}\right) \cdot \hat{e}\left(u \cdot P, R_{I D}+h \cdot P_{p u b}\right) \\
& =\hat{e}\left(U_{1}, P_{I D}\right) \cdot \hat{e}\left(U_{2}, R_{I D}+h \cdot P_{p u b}\right) \\
& =\hat{e}\left(P_{I D}, U_{1}\right) \cdot \hat{e}\left(R_{I D}+h \cdot P_{p u b}, U_{2}\right) .
\end{aligned}
$$

- Forgery: Assume that the adversary $\mathcal{A}$ forges a valid signature tuple $\left(m^{*}, \sigma^{*}, I D^{*}\right)$ under the public key $P K_{I D^{*}}$. Here $P K_{I D^{*}}$ is the original public key without replacement. First, the challenge $C$ obtains the corresponding tuple $\left(m^{*}, I D^{*}, P K_{I D^{*}}, u\right.$, $U_{2}$, coin) in the list $L_{2}$. We discuss three cases.

1. If $I D^{*} \neq I D^{\prime}$, the challenger $C$ returns failure and terminates.

2. If $I D^{*}=I D^{\prime}$ and coin $=0$, the challenger $C$ returns failure and terminates.

3. If $I D^{*}=I D^{\prime}$ and coin=1, the challenger $C$ uses the forgery signature $\sigma^{*}$ to solve the $\mathrm{CDH}$ problem as follows. Since $\sigma^{*}$ is valid,

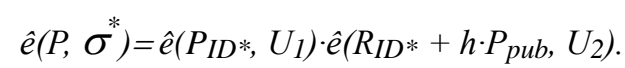

By properties of bilinear pairings,

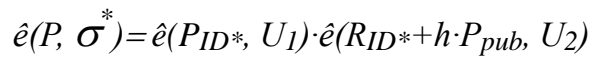

$$
\begin{aligned}
& =\hat{e}\left(P_{I D} *, w \cdot P\right) \cdot \hat{e}\left(r_{I D} * P+h \cdot a P, u \cdot b P\right) \\
& =\hat{e}\left(w \cdot P_{I D} * P\right) \cdot \hat{e}\left(r_{I D} * P, u \cdot b P\right) \cdot \hat{e}(h \cdot a P, u \cdot b P) \\
& =\hat{e}\left(w \cdot P_{I D}, P\right) \cdot \hat{e}\left(r_{I D} * \cdot u \cdot b P, P\right) \cdot \hat{e}(h u \cdot a b P, P) \\
& =\hat{e}\left(w \cdot P_{I D} *+r_{I D} * U_{2}+h u \cdot a b P, P\right) \\
& =\hat{e}\left(P, w \cdot P_{I D} *+r_{I D} * U_{2}+h u \cdot a b P\right) \text {. }
\end{aligned}
$$

So, we have $\sigma^{*}=w \cdot P I D^{*}+r I D^{*} \cdot U_{2}+h u \cdot a b P$. Then, the challenger $C$ outputs $\left(\sigma^{*}-w \cdot P I D^{*-}-r I D^{*} U_{2}\right) / h u$ as the solution $a b P$ of the $\mathrm{CDH}$ problem. Finally, we calculate the probability that $C$ does not abort during the simulation. The probability that it does not abort during the Certificate generation query and the Forgery phases are $\left(1-1 / q_{\mathrm{c}}\right)$ and $\delta / q_{\mathrm{c}}$, respectively. In 
addition, in the Sign query phase, the probability that it does not abort is $(1-\delta / q)^{q_{s}} \geq(1-c \delta)^{q_{s}}$. Therefore, the probability that $C$ does not abort is $(1-1 / q)(1-\delta)^{q_{s}}$ $\delta / q$. This value can be maximized at $\delta_{o p t}=1 /\left(q_{s}+1\right)$. Hence, if the adversary $\mathcal{A}$ has a non-negligible advantage $\varepsilon$ to break the proposed SCBS scheme, the challenger $C$ has a non-negligible advantage

$$
\varepsilon^{\prime} \geq\left(1-\frac{1}{q_{c}}\right)\left(1-\frac{1}{1+q_{s}}\right)^{q_{s}}\left(\frac{1}{\left(q_{c}\left(1+q_{s}\right)\right)}\right) \varepsilon
$$

to solve the $\mathrm{CDH}$ problem. Finally, to answer queries in the simulation game above, the required computation time is $\tau^{\prime}=\tau+O\left(q_{c}+q_{s}+q_{u}+q_{1}+q_{2}\right) \tau_{1}$, where $\tau_{1}$ is the time to perform a scalar multiplication in $G$. $\square$

Theorem 2. Suppose that the hash functions $H_{1}$ and $\mathrm{H}_{2}$ are random oracles. Assume that a Type II UF-SCBS-ACM adversary $\mathcal{A}$ can break the proposed SCBS scheme with non-negligible advantage

within running time $\tau$. Then, there exists an algorithm $C$ to solve the $\mathrm{CDH}$ problem with a non-negligible advantage

$$
\varepsilon^{\prime} \geq\left(1-\frac{1}{q_{u}}\right)^{q_{\text {cor }}}\left(1-\frac{1}{1+q_{s}}\right)^{q_{s}}\left(\frac{1}{q_{u}\left(1+q_{s}\right)}\right) \varepsilon
$$

within running time $\tau^{\prime}=\tau+O\left(q_{s}+q_{u}+q_{1}+q_{2}\right) \tau_{1}$, where $\tau_{1}$ is the time to perform a scalar multiplication in $G$. Also, $q_{c o r}, q_{s}, q_{u}, q_{1}$, and $q_{2}$ denote, respectively, the maximal numbers of $\mathcal{A}$ 's queries to the Corruption oracle, the Sign oracle, the User key generation oracle, the random oracle $H_{1}$, and the random oracle $\mathrm{H}_{2}$.

Proof. Assume that an algorithm $C$ is given a random instance $(P, a P, b P)$ of the $\mathrm{CDH}$ problem, where $P$, $a P, b P \in G$ with unknown $a, b \in Z_{q}{ }^{*}$. Let $J=a b P$ be the solution of the $\mathrm{CDH}$ problem. The algorithm $C$ finds $J$ by interacting with the adversary $\mathcal{A}$ as follows.

- Setup. The challenger $C$ creates a list of public parameters $P P=\left\{G, G T, q, \hat{e}, P, P_{p u b}, f, H_{1}, H_{2}\right\}$ and $P_{p u b}=s \cdot P$, where $s$ is the master secret key. The challenger $C$ gives $\mathcal{A}$ the public parameters $P P$ and $s$. Here, the hash functions $f, H_{1}$ and $H_{2}$ are random oracles controlled by the challenger $C$. To be consistent and collision-free, the challenger $C$ maintains three initially empty lists $L_{f}, L_{1}$ and $L_{2}$ to record the responses of the hash functions. Since the adversary $\mathcal{A}$ knows the master secret key $s$, it can compute the certificate $C_{I D}$ and know all users' public keys so that it does not need to issue the certificate generation query.

- Queries: The challenger $C$ first randomly chooses $t \in\left[1, q_{u}\right]$ and lets $I D^{\prime}$ denote the target identity of the $t$-th query to the User key generation oracle.
The challenger $C$ answers the queries issued by $\mathcal{A}$ as follows.

- User key generation (ID): C maintains an initially empty list $P^{\text {list }}$ of tuples $\left(I D, P I D, S_{I D}\right)$. If $I D$ 's corresponding tuple $\left(I D, P I D, S_{I D}\right)$ already exists in $P^{\text {list }}, C$ returns $P I D$ as answer. Otherwise, we split into two cases. If $I D \neq I D^{\prime}, C$ randomly chooses a value $S_{I D} \in Z_{q}{ }^{*}$ and computes $P_{I D}=$ $S_{I D} \cdot P$. If $I D=I D^{\prime}, C$ sets $P I D=a P$ and $S_{I D}=\perp$. In both cases, $C$ adds (ID, $\left.P I D, S_{I D}\right)$ to $P^{\text {list }}$, and returns $P_{I D}$ to the adversary $\mathcal{A}$.

- $\quad f\left(I D, P_{I D}, R_{I D}\right)$ : As the $f\left(I D, P_{I D}, R_{I D}\right)$ query in Theorem 1.

- $\quad H_{1}\left(m, I D, P K_{I D}\right): C$ maintains an initially empty list $L_{1}$ of tuples $\left(m, I D, P K_{I D}, w, U_{1}\right.$, coin). If there exists a tuple $\left(m, I D, P K_{I D}, w, U_{1}\right.$, coin $)$ in $L_{1}$ related to the query $H_{1}\left(m, I D, P K_{I D}\right), C$ returns $U_{1}$ to the adversary $\mathcal{A}$. Otherwise, $C$ first randomly chooses $w \in Z_{q}^{*}$. Then, if $I D \neq I D^{\prime}, C$ sets $U_{1}=w \cdot P$ and coin $=0$. If $I D=I D^{\prime}, C$ tosses a coin $\in\{0,1\}$ with $\operatorname{Pr}[\operatorname{coin}=1]=\delta$ for some $\delta$ that will be determined later. Moreover, $C$ sets $U_{1}=$ $w \cdot P$ if $\operatorname{coin}=0$, and $U_{1}=w \cdot b P$ if coin $=1$. Finally, $C$ returns the corresponding $U_{1}$ to $\mathcal{A}$ and adds ( $m$, $I D, P K_{I D}, w, U_{1}$, coin) to $L_{1}$.

- $H_{2}\left(m, I D, P_{I D}, R_{I D}\right): C$ maintains an initially empty list $L_{2}$ of tuples $\left(m, I D, P K_{I D}, u, U_{2}\right)$. If there exists a tuple $\left(m, I D, P K_{I D}, u, U_{2}\right)$ in $L_{2}$ related to the query $H_{2}\left(m, I D, P_{I D}, R_{I D}\right), C$ returns $U_{2}$ to $\mathcal{A}$. Otherwise, $C$ randomly chooses $u$ $\in Z_{q}^{*}$, sets $U_{2}=u \cdot P$, returns $U_{2}$ to $\mathcal{A}$, and adds ( $\left.m, I D, P K_{I D}, u, U_{2}\right)$ to $L_{2}$.

- Corruption (ID): If ID's corresponding tuple (ID, $\left.P I D, S_{I D}\right)$ already exists in the list $P^{\text {list }}, C$ returns

- $\quad S_{I D}$ as answer. Otherwise, $C$ issues User key generation query on $I D$ to obtain the associated $S_{I D}$. Finally, $C$ returns $S_{I D}$ to the adversary $\mathcal{A}$ if $I D \neq I D^{\prime}$, and returns failure and terminates, otherwise.

- $\quad$ Public key replace (ID, $\left.P K^{\prime} I D\right)$ : The adversary $A$ chooses a new public key $P K^{\prime} I D=\left(P^{\prime} I D, R^{\prime} I D\right)$ of a user with identity $I D$ and sends it to the challenger $C$. Then $C$ replaces the corresponding tuple $\left(I D, P_{I D}, S_{I D}\right)$ in $P^{\text {list }}$ with the new tuple $(I D$, $\left.P^{\prime} I D, \perp\right)$.

- $\quad \operatorname{Sign}(m, I D)$ : When receiving a signature query on $(m, I D)$ under the public key $P K_{I D}=\left(P_{I D}\right.$, $R_{I D}$ ), the challenger $C$ first issues the $H_{2}$ query with $\left(m, I D, P K_{I D}\right)$ and obtains the 
corresponding tuple $\left(m, I D, P K_{I D}, w, U_{1}\right.$, coin $)$ in $L_{1}$. The challenger $C$ discusses two cases.

1. If $I D=I D^{\prime}$ and $\operatorname{coin}=1, C$ returns failure and terminates.

2. Otherwise, $C$ chooses two random elements $h, u \in Z_{q}^{*}$ and computes the signature $\sigma=w \cdot P_{I D}+u \cdot R_{I D}+u \cdot h \cdot P_{p u b}$. Then, $C$ adds $\left(I D, P_{I D}, R_{I D}, h\right)$ in $L_{f}$ and $\left(m, I D, P K_{I D}, u\right.$, $\left.U_{2}\right)$ in $L_{2}$, and returns the signature $\sigma$. The signature $\sigma$ is valid because it can pass the verification, as described in the proof of Theorem 1

- Forgery: Assume that the adversary $\mathcal{A}$ forges a valid signature tuple $\left(M^{*}, \sigma^{*}, I D^{*}\right)$ under the public key $P K_{I D^{*}}=\left(P_{I D^{*}}, R_{I D^{*}}\right)$. First, the challenger $C$ obtains the corresponding tuple $\left(m^{*}, I D^{*}, P K_{I D^{*}}, w\right.$, $U_{1}$, coin) in the list $L_{1}$. We discuss three cases.

1. If $I D^{*} \neq I D^{\prime}$, the challenger $C$ returns failure and terminates.

2. If $I D^{*}=I D^{\prime}$ and $\operatorname{coin}=0$, the challenger $C$ returns failure and terminates.

3. If $I D^{*}=I D^{\prime}$ and coin=1, the challenger $C$ uses the forgery signature $\sigma^{*}$ to solve the $\mathrm{CDH}$ problem as follows. Since $\sigma^{*}$ is valid,

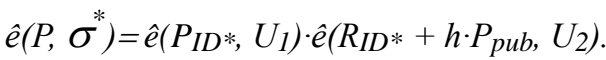

By properties of bilinear pairings,

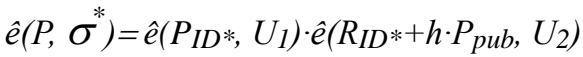

$=\hat{e}(a P, w \cdot b P) \cdot \hat{e}\left(R_{I D} *+h \cdot s \cdot P, u \cdot P\right)$

$=\hat{e}(w \cdot a b P, P) \cdot \hat{e}\left(u \cdot R_{I D} *+u \cdot h \cdot s \cdot P, P\right)$

$=\hat{e}\left(w \cdot a b P+u \cdot R_{I D} *+u \cdot h \cdot s \cdot P, P\right)$

$=\hat{e}\left(P, w \cdot a b P+u \cdot R_{I D} *+u \cdot h \cdot s \cdot P\right)$.

So, we have $\sigma^{*}=w \cdot a b P+u \cdot R_{I D} *+u \cdot h \cdot s \cdot P$. Then, the

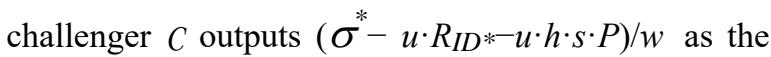
solution $a b P$ of the $\mathrm{CDH}$ problem. Finally, we calculate the probability that $C$ does not abort during the simulation. The probability that it does not abort during the corruption query phase is (1$\left.1 / q_{u}\right)_{\text {cor }}^{q}$ and the Forgery phase is $\delta / q_{u}$. In addition, in the Sign query phase, the probability that it does not abort is $(1-\delta / q)^{q_{s}} \geq(1-\delta)^{q_{s}}$. Therefore, the probability that $C$ does not abort is $\left(1-1 / q_{u}\right)^{q_{\text {cor }}}(1-$ $\delta)^{q_{s}} \delta / q_{u}$. This value can be maximized at $\delta_{\text {opt }}=1 /\left(q_{s}\right.$ $+1)$. Hence, if the adversary $\mathcal{A}$ has a non-negligible advantage $\varepsilon$ to break the proposed SCBS scheme, the challenger $C$ has a non-negligible advantage

$$
\varepsilon^{\prime} \geq\left(1-\frac{1}{q_{u}}\right)^{q_{\text {cor }}}\left(1-\frac{1}{1+q_{s}}\right)^{q_{s}}\left(\frac{1}{q_{u}\left(1+q_{s}\right)}\right) \varepsilon
$$

to solve the $\mathrm{CDH}$ problem. Finally, for answering queries in the simulation game above, the required computation time is $\tau^{\prime}=\tau+O\left(q_{s}+q_{u}+q_{1}+q_{2}\right) \tau_{1}$, where $\tau_{1}$ is the time to perform a scalar multiplication in G. $\square$

\section{Performance comparisons and discussions}

In the following, we first define several timeconsuming operations.

- $T_{p}$ : the time of executing a bilinear pairing operation $\hat{e}: G \times G \rightarrow G_{T}$;

- $T_{m}$ : the time of executing a scalar multiplication in $G$, an exponentiation in $G_{T}$ or an exponentiation operation in $Z_{q}{ }^{*}$;

- $T_{h}$ : the time of executing a map-to-point hash function.

In the following, Table 2 lists the comparisons among Zhang's CBS scheme [28], Liu et al.'s SCBS scheme [30], Li et al.'s SCBS scheme [24] and our SCBS scheme in terms of signature size,

the computational costs of signing and verification, and security. For the signature size, Zhang's scheme requires two elements in $G$ while Liu et al.'s, Li et al.'s and our schemes require only one element in $G$.

For the computational cost of the signing and verification phases, our scheme is better than Zhang's scheme, but requires more operations than Liu et al.'s and Li et al.'s schemes. However, both Liu et al.'s and $\mathrm{Li}$ et al.'s schemes suffer from the attacks of Type I adversary under an accredited security model. According to Table 2, our scheme is provably secure while retaining efficiency.

Table 2. Comparisons between the previously proposed CBS and SCBS schemes and ours

\begin{tabular}{ccccc}
\hline & $\begin{array}{c}\text { Zhang's CBS scheme } \\
{[\mathbf{2 8}]}\end{array}$ & $\begin{array}{c}\text { Liu et al.'s SCBS } \\
\text { scheme [30] }\end{array}$ & $\begin{array}{c}\text { Li et al.'s SCBS scheme } \\
{[\mathbf{2 4}]}\end{array}$ & $\begin{array}{c}\text { Our SCBS } \\
\text { scheme }\end{array}$ \\
\hline Signature size & 2 & 1 & 1 & 1 \\
\hline $\begin{array}{c}\text { Computation cost of signing } \\
\begin{array}{c}\text { Computation cost of } \\
\text { verification }\end{array}\end{array}$ & $5 \mathrm{Tm}$ & $\mathrm{Tm}+\mathrm{Th}$ & $\mathrm{Tm}+\mathrm{Th}$ & $2 \mathrm{Tm}+2 \mathrm{Th}$ \\
\hline Type I attack & UF-CBS-ACM & Existing attack [29] & $\begin{array}{c}\text { Existing attack (Section } \\
4)\end{array}$ & UF-SCBS-ACM \\
\hline Type II attack & UF-CBS-ACM & UF-SCBS-ACM & UF-SCBS-ACM & UF-SCBS-ACM \\
\hline
\end{tabular}




\section{Conclusions}

In this article, we demonstrate that $\mathrm{Li}$ et al.'s SCBS scheme is insecure against the attacks of Type I adversary under an accredited security model. We also propose the first provably secure SCBS scheme in the random oracle model. In the accredited security model, the adversary is allowed to issue the Sign query even though the challenger does not hold the corresponding secret key and the certificate of the user. This is the strongest capability that an adversary can possess in SCBS schemes. Moreover, due to the short signature length, our SCBS scheme is well suited for low-bandwidth communication environments with high-level security.

\section{Acknowledgements}

The authors would like to appreciate anonymous referees for their valuable comments and constructive suggestions. This research was partially supported by Ministry of Science and Technology, Taiwan, R.O.C., under grant no. MOST103 -2221-E018-022-MY2.

\section{References}

[1] T. ElGamal. A public key cryptosystem and a signature scheme based on discrete logarithms. In: Proc. of Crypto'84, LNCS, 196, 1984, pp. 10-18.

[2] R. L. Rivest, A. Shamir, L. Adleman. A method for obtaining digital signatures and public-key cryptosystems. Commun. of ACM, 1978, Vol. 21, No. 2, $120-126$.

[3] A. Shamir. Identity-based cryptosystems and signature schemes. In: Proc. of Crypto'84, LNCS, 196, 1984, pp. 47-53.

[4] D. Boneh, M. Franklin. Identity-based encryption from the Weil pairing. In: Proc. of. Crypto'01, LNCS, 2139, 2001, pp. 213-229.

[5] S. S. Al-Riyami, K. G. Paterson. Certificateless public key cryptography. In: Proc. of ASIACRYPT'03, LNCS, 2894, 2003, pp. 452-473.

[6] Y. M. Tseng, T. T. Tsai. Efficient revocable ID-based encryption with a public channel.

[7] Computer Journal, 2012, Vol. 55, No. 4, 475-486.

[8] T. T. Tsai, Y.M. Tseng. Revocable certificateless public key encryption. IEEE Systems Journal, 2015, Vol. 9, No. 3, 824-833.

[9] Y. H. Hung, T. T. Tsai, Y. M. Tseng, S. S. Huang. Strongly secure revocable ID-based signature without random oracles. Information Technology and Control, 2014, Vol. 43, No. 3, 264276.

[10] T. T. Tsai, S. S. Huang, Y.M. Tseng. Secure certificateless signature with revocation in the standard model. Mathematical Problems in Engineering, 2014, Vol. 2014, Article ID 728591.

[11] C. Gentry. Certificate-based encryption and the certificate revocation problem. In: Proc. of Eurorypt'03, LNCS, 2656, 2003, pp. 272-293.
[12] A. Fiat, A. Shamir. How to prove yourself: practical solutions to identification and signature Problems. In: Proc. of Crypto'86, LNCS, 263, 1987, pp. 186-194.

[13] K. Kurosawa, S. Heng. From digital signature to ID-based identification/signature. In: Proc. of PKC'04, LNCS, 2947, 2004, pp. 248-261.

[14] [Y. M. Tseng, T.Y. Wu, J.D. Wu. A pairing-based user authentication scheme for wireless clients with smart cards. Informatica, 2008, Vol. 19, No. 2, 285-302.

[15] D. He, N. Kumar, N. Chilamkurti. A secure temporalcredential-based mutual authentication and key agreement scheme with pseudo identity for wireless sensor networks. Information Sciences, 2015, Vol. 321, 263-277.

[16] D. He, S. Zeadally. Authentication protocol for an ambient assisted living system. IEEE Communications Magazine, 2015, Vol. 53, No. 1, 71-77.

[17] K. Barr, K. Asanovic. Energy aware lossless data compression. ACM Transactions on Computer Systems, 2006, Vol. 24, No. 3, 250-291.

[18] D. Boneh, B. Lynn, H. Shacham. Short signatures from the Weil pairing. In: Proc. of ASIACRYPT'01, LNCS, 2248, 2001, pp. 514-532.

[19] D. Boneh, X. Boyen. Short signatures without random oracles. In: Proc. of EUROCRYPT'04, LNCS, 3027, 2004, pp. 56-73.

[20] F. Zhang, X. Chen, W. Susilo, Y. Mu. A new short signature scheme without random oracles from bilinear pairings. IACR Cryptology ePrint Archive, 2005, Article 386.

[21] K. Y. Choi, J.H. Park, D.H. Lee. A new provably secure certificateless short signature scheme. Computers and Mathematics with Applications, 2011, Vol. 61, No. 7, 1760-1768.

[22] R. Tso, X. Huang, W. Susilo. Strongly secure certificateless short signatures. Journal of Systems and Software, 2012, Vol. 85, No. 6, 1409-1417.

[23] Y. C. Chen, R. Tso, W. Susilo, X. Huang, G. Horng. Certificateless signatures: structural extensions of security models and new provably secure schemes. IACR Cryptology ePrint Archive, 2013, Article 193.

[24] D. He, B. Huang, J. Chen. New certificateless short signature scheme. IET Information Security, 2013, Vol. 7, No. 2, 113-117.

[25] J. Li, X. Huang, Y. Zhang, L. Xu. An efficient short certificate-based signature scheme. Journal of Systems and Software, 2012, Vol. 85, No. 2, 314-322.

[26] B. G. Kang, J. H. Park, S. G. Hahn. A Certificatebased signature scheme. In: Proc. of CT-RSA'04, LNCS, 2964, 2004, pp. 99-111.

[27] J. Li, X. Huang, Y. Mu, W. Susilo, Q. Wu. Certificate-based signature: security model and efficient construction. In: Proc. of EuroPKI' 07, LNCS, 4582, 2007, pp. 110-125.

[28] J. K. Liu, J. Baek, W. Susilo, J. Zhou. Certificatebased signature scheme without pairings or random oracles. In: Proc. of ISC'08, LNCS, 5222, 2008, pp. 285-297.

[29] J. Zhang. On the security of a certificate-based signature scheme and its improvement with pairings. In: Proc. of ISPEC'09, LNCS, 5451, 2009, pp. 47-58.

[30] W. Wu, Y. Mu, W. Susilo, X. Huang. Certificatebased signatures revisited. Journal of Universal Computer Science, 2009, Vol. 15, No. 8, 1659-1684. 
[31] J. K. Liu, F. Bao, J. Zhou. Short and efficient certificate-based signature. In: Proc. of NETWORKING 2011 Workshops, LNCS, 6827, 2011, pp. $167-178$.

[32] L. Cheng, Y. Xiao, G. Wang. Cryptanalysis of a certificate-based on signature scheme. Procedia Engineering, 2012, Vol. 29, 2821-2825.
[33] M. Bellare, P. Rogaway. Random oracles are practical: a paradigm for designing efficient protocols. In: Proc. of 1st ACM conference on Computer and Communications Security, 1993, pp. 6273

[34] R. Canetti, O. Goldreich, S. Halevi. The random oracle methodology, revisited. J. ACM, 2004, Vol. 51, No. 4, 557-594.

Received July 2015. 